\title{
Learning Capacity in Simulated Virtual Neurological Procedures
}

\author{
Mattia Samuel Mancosu \\ Department of Computer \\ Science, Liverpool John \\ Moores University \\ Brownlow Hill \\ L3 5UG, Liverpool, \\ United Kingdom \\ m.s.mancosu@2019.ljmu.ac.uk
}

\author{
Silvester Czanner \\ Department of Computer \\ Science, Liverpool John \\ Moores University \\ Brownlow Hill \\ L3 5UG, Liverpool, \\ United Kingdom \\ s.czanner@ljmu.ac.uk
}

\author{
Martin Punter \\ Senior Medical Officer \\ Capital and Coast District \\ Health Board \\ 69 Riddiford Street \\ Wellington, New Zealand \\ martin.punter@ccdhb.org.nz
}

\begin{abstract}
In recent years, the use of Virtual Reality (VR) has skyrocketed in many fields. From videogames to education, the popularity of this technology is well known also thanks to its affordability and the growing number of available content. An immersive approach and the correct use of gamification have been proven to be valuable tools for learning in different fields. There are many examples of the adoption of VR application also in the healthcare setting not only for learning purposes but also for patients' rehabilitation. This paper is intended to list some existing works and then present our project for an immersive serious game with the aim of enhancing the training of healthcare workers for the assessment of stroke patients.
\end{abstract}

\section{Keywords}

Virtual Reality, Serious Games, Gamification, Healthcare, Stroke, Evaluation, Assessment, Training

\section{INTRODUCTION}

Real-life simulation is frequently used in clinical practice for training in the United Kingdom and further afield. One of the most successful programmes is with simulation training for Advanced Life Support where regular highly structured practice improves team performance. Simulation training is also taking on an increasing role in the training of medical students, junior doctors, and Allied Health Professionals in day to day work, whether for specific procedures or specific team practices. At Salford Royal NHS Foundation Trust (SRFT) has been recently introduced real-time simulation training for the stroke team, using a mannequin and running through clinical scenarios relating to acute stroke treatment.

It can be increasingly a challenge, however, in the $\mathrm{Na}$ tional Health Service, to find appropriately convenient times to bring whole teams together in one place for such training and the opportunity for VR training may improve staff confidence in advance of real-time simulation training, or clinical practice, and would allow training to be delivered simultaneously across multiple sites. Whilst serious games or VR have been described as used in the rehabilitation of stroke patients (Cochrane review 2015, Laver et al) to our knowledge no publications are describing the use of VR as a tool for systematic training of healthcare professionals involved in the care of patients with acute stroke.

\section{VIRTUAL REALITY}

For Soysa et al. Virtual Reality is intended as a HumanComputer interface where a computer system generates a three dimensional, sensory, immersing environment that responds in an interactive way to the behaviour of the user [1]. The purpose of their research was to create a framework for VR and Virtual Instrumentation for educational purposes, performing an evaluation of an application based on the response of users to haptic feedback. Until a few years ago VR was not immediately associated with the immersive part of the experience. Only recently have head-mounted displays (HMD) become increasingly popular and led to a natural association with VR. Previously, many VR projects orbited around the concept of Collaborative Virtual Environments (CVE), defined by Normand et al. as the use of natural spatial metaphors, together with the integration of participants and data within the same and common spatial frame of reference [2]. For example, Nakai et al. realised a network infrastructure for peer to peer distributed computation on a collaborative virtual envi-

\footnotetext{
Permission to make digital or hard copies of all or part of this work for personal or classroom use is granted without fee provided that copies are not made or distributed for profit or commercial advantage and that copies bear this notice and the full citation on the first page. To copy otherwise, or republish, to post on servers or to redistribute to lists, requires prior specific permission and/or a fee.
} 
ronment [3]. With the proper use of Level Of Details function for $3 \mathrm{D}$ elements and the correct distribution of the contents within the nodes of the peer to peer network, they achieve a good level of usability and scalability of the CVE system. There are many articles concerning rules and guidelines for the proper use of VR and CVE. Levels of detail is considered a crucial feature when designing a VR application also by Seo and $\mathrm{Kim}$, as they help cope with problems related to bigger environments or incrementally complex scenes [4] In 1999 Noll et al. described the interaction between users inside a shared virtual environment and how this interaction could enhance their work. This research covered also the areas of distributed simulation, agents in virtual environments, and networking for large-scale virtual environments [5]. Kuutti et al. stated that virtual prototypes can be used for usability testing and the quality of environment and models are crucial for the success of the testing [6]. In fact, Yen and NG also showed that Virtual Objects are successful tools for Virtual Environments if they are properly implemented and can be far more useful than simple images [7]. Furthermore, a study conducted by Drettakis et al. illustrated an evaluation approach for real-world applications in the context of an urban planning project, demonstrating the relevance of both audio and visual realism, such as 3D sound, shadows, sun coverage, vegetation, and crowds [8]. Many evaluation guidelines on VR application for multi-disciplinary activities are addressed by Livatino and Koffel [9]. Their two pilot studies successfully proved the correctness and the reasonableness of those guidelines for students and non-experts. More recently, Bouville et al. presented a framework for developing an interactive and collaborative virtual environment. Their system is based on a relation engine module and a collaborative interaction engine module. The first one manages the relations between the objects of the environment and the collaborative interaction engine manages how users can collaboratively control objects [10].

\subsection{Immersive Virtual Reality}

The immersive approach to Virtual Reality extended the possible applications, the type of interactions and the quality of the experience. Corrêa et al. researched how a realistic $3 \mathrm{D}$ environment in conjunction with immersive VR is perceived. Performing an evaluation on interactive archaeometry, they concluded that it is possible to represent VE realistically as the real one [11]. The use of HMD has led to multiple new interaction possibilities, reflected in the number of new studies concerning movement, user interface and usability. For example, Gebhardt et al. performed a study focused on different entry selection methods for hierarchical pie menus. They tested the use of input devices different from the standard keyboard and mouse, and they con- cluded that the pick-ray-based entry selection metaphor is the most suitable for this case of study [12]. In 2014 Emma et al. described the nature of usability issues in immersive CVE. The evaluation is performed inside a framework where errors observed are interpreted as rule-based mistakes. The errors are mainly connected with tactual and/or postural issues. In fact, the lack of tactual feedback when touching an object and the sitting position of the participants had contributed to thwarting the realism of the actions [13].

\section{SERIOUS GAMES}

VR and teaching is a well known successful combination, there are countless educational and industrial application instances. For the field of Mechanical Engineering, Muller et al. presented a preliminary study on the use of consumer-grade VR devices, describing how to design an immersive learning game and how to evaluate the user experience. The first exploratory experiment showed a high interest from teachers and students, laying the foundations for future improvement of the training in the Mechanical Engineering field [14]. Concerning learning games and safety, in 2017 Zhang et al. designed a framework for fire safety education implemented according to the combination of relevant fire safety education theory and immersive VR technology. Using an HTC VIVE helmet equipment for the user test, they again proved the effectiveness of this technology to help students learn fire safety knowledge, master fire safety skills and improve fire safety education effect [15]. Another example of immersive experience in safety education is the game developed by Vega et al. in 2017. The design is focused on a controlled and engaging environment to practice and learn how to safely control a campfire. They received positive feedback in many competitions, although they still have to perform a proper evaluation of the game [16]. On the subject, Andrès et al.affirmed that "to demonstrate the effectiveness of a serious game, a holistic evaluation process is required". Their first objective was to evaluate a serious game called Timemesh and to show the importance of adequate evaluation process [17]. An in-depth exemplar designed to illustrate how VR can be used to promote aspects of Deeper Learning[18] for high school students is provided in a paper by Southgate. The study shows how VR can be used for selfdirected, collaborative and imaginative learning [19]. Zhang et al. explored the possible learning outcomes derived by different the level of interaction in a single VR learning application. Although they were not able to find a significant correlation between level of interaction and learning outcome, the interaction has been proven to be a key factor for engagement and their data suggests that "interactivity-triggered concept visualisation and animation are more effective for learning than automated visualisation and animation" [20]. 


\subsection{Serious Games in Healthcare}

Games are often used for training healthcare workers and during patients rehabilitation. The proper application of gamification techniques can be crucial to both categories. In this regard, Ribeiro et al. in 2019 presented a system to practice dental anaesthesia techniques. Their serious games allow the user to practice with or without tactile or visual aids thanks to a haptic interface [21]. Also, Ushaw et al. proved the help given by serious games for health. Their game for measuring the stereoacuity of young children has been tested by an initial user group and was proven successful, although a testing protocol and rapid analysis of the data is needed [22]. In this paper, our main research objective is immersive VR and serious games in healthcare, more specifically in the rapid assessment and treatment of acute stroke, are relatively common emergency neurological disease. In 2006 Kaul combined gaming technology with brain wave research to create a gaming environment that functions as a neurological learning tool, explaining that mental states can be focused and the adoption of a content-driven environment can help the ability to perceive information [23]. Alankus et al. cited the importance of multiple repetitions of motion with the affected limbs in patients paralysed by strokes. Considering the high rate of unmotivated patients, home-based stroke rehabilitation games may help motivate stroke patients to perform the necessary exercises to recover. Starting from the previous considerations, Alankus et al. developed several games for coping with different necessities for rehabilitation such impossibility of the use of hands and the support for multiple methods of user input [24]. Those games are not realised using VR techniques, however, they proved their value from a therapeutic perspective even with a limited number of tested patients. Choi, on the other hand, presents the application of VR technology in wound care training. The proposed application simulates the steps for changing a wound dressing with the help of visual cues and texts. Although this study is not concluded yet and comprehensive evaluation is still needed, the system has the promising benefit of facilitating self-learning at own pace while saving the cost of wound dressing materials [25].

\section{GAMIFICATION}

Serious Games share with Gamification a similar intent, which is learning having fun to improve the effectiveness of teaching. Gamification, however, is the application of game elements (such as badges and leader boards) to other areas of activity to increase effectiveness and engagement. We are then talking about general-purpose applications instead of games, which have fun and entertainment as primary objectives. Marczewski defines Gamification as "the use of game design metaphors to create more game-like and engaging experiences."[26] For instance, Pastushenko et al. developed a tool for the conceptual learning of electric and electronic circuits. In this case, the student has to modify some values on the proposed circuits in order to obtain the correct voltage across the given components and let the game character "surf" to the next circuit [27]. The research proposed by Coull et al. makes use of Gamification for developing an application capable of simulate cybercrimes and provide training to police officers. A virtual environment is adopted to host a scenario where the officers can explore a specific crime scene and, by inspecting elements such as smartphones and laptops, give feedback with the appropriate course of action. The results have demonstrated the potential of this type of training considering also a significantly reduced cost with respect to expensive classroom-based sessions [28].

\subsection{Gamification in Healthcare}

Gamification is also a tool used for healthcare applications. Jacobs et al. designed a game for stroke survivors aimed to make the rehabilitation process more enjoyable. Their application involves the use of common objects and a multi-touch screen to perform armhand exercises in order to restore physical and cognitive capabilities. The results of the test conducted on two patients showed promising therapy results with a noticeable improvement of their arm-hand functionality [29]. An online game for medical education on insulin therapy has been objective of study for Diehl et al. The game is a tool for the education of doctors in the treatment of patients with diabetes mellitus and Diehl et al. collected results from 134 of them after 21 days of interaction with the game. Two groups were formed to study the difference between who played the game (game group) and the control group. The absolute increase in competence score and the frequency of subjects who achieved a high score in the post-intervention assessment was considerably higher in the game group than in the control group [30]. Vascular Invaders is a web-based study aid with game features adopted by Gauthier et al. to perform a comparative study between regular learning and eLearning on a group of medical anatomy students. Their results showed the necessity for a more exploratory study of the subject considering the promising results [31]. Boada et al. analysed the impact of using LISSA, a game to complement CPR teaching, after theory introduction and before laboratory practice on 109 nursing undergraduate students. Even then, the use of Gamification helped to engage the students and better results were obtained with respect to students that only read theory material [32]. 


\section{SIMULATED NEUROLOGICAL TRAINING}

Neurological procedures are very complex. It is almost impossible to simulate the whole procedure for several reasons. Even there is a guidance and exacts assessment instructions for the neurologists, each procedure is different in terms of patients medical conditions. Based on neurological advice we have identified a clearly defined project, of manageable size and complexity that established and rigorously test the necessary technical capabilities in an experimental setting.Real-life simulated training is well established in certain medical specialties (typically emergency department, CPR training, ICU medicine) and is being increasingly used in other specialites. There is a a great deal of interest in the use of simulation for the assessment and treatment of acute stroke as it is a highly protocolised and time sensitive treatment and with widely available stroke specialist teams, developing efficient team working improves outcomes for patients. The overall goal in this project was to design and develop a virtual stroke simulator with software support to use for simulation of acute stroke assessment and treatment. The aim is educating healthcare professional involved in the management of acute stroke and provide insight for young and elderly people on the effects and symptoms of a stroke and what to expect in the hospital setting. The proposed virtual stroke simulator is built on a spectrum of information acquired by a detailed literature search, input from clinical studies, and through discussions with clinical neurologists. We are using a standardised approach to build the simulator based on international treatment guidelines and structured rating scales of stroke severity and inclusion/exclusion criteria for the various treatments of stroke.

One of the main issues with the training for healthcare professionals is availability and time restriction. It can be a challenge, particularly in the modern healthcare setting, to gather a learning group in one place for training. Therefore being able to access, either individually or as a team, an online simulation training package could offer a solution. In the setting of busy stroke units, the opportunity to train in a virtual environment with one or more team members participating in a VR game would provide enhances opportunities for practice and refining teamwork. Furthermore, the standard training procedure involves the use of mannequins which can be suitable for helping with only a few of the many steps needed for assessing a real stroke case. For example, facial palsy, aphasia and limb ataxia cannot be shown using a mannequin and usually, they are simulated by a volunteer. By developing such simulator we aim to improve the effectiveness of the training.

\subsection{Acute Stroke Assessment}

A stroke is a medical condition where the blood supply of a part of the brain is cut off and it is usually diagnosed by doing physical tests and studying images of the brain produced during a scan [33]. Due to the complexity of the assessment, our simulation is limited to the assessment of the severity grade of the stroke and it will be based on physical movement, facial expressions, speech and other actions necessary for the evaluation but not currently possible to replicate using a mannequin. We decided to simulate the National Institutes of Health Stroke Scale (NIHSS) procedure. "The NIHSS is a 15-item neurologic examination stroke scale used to evaluate the effect of acute cerebral infarction on the levels of consciousness, language, neglect, visual-field loss, extraocular movement, motor strength, ataxia, dysarthria, and sensory loss. A trained observer rates the patent's ability to answer questions and perform activities. Ratings for each item are scored with 3 to 5 grades with 0 as normal, and there is an allowance for untestable items. The single patient assessment requires less than 10 minutes to complete. The evaluation of stroke severity depends upon the ability of the observer to accurately and consistently assess the patient."[34]

\subsection{Pilot Studies}

The predecessors of our project were two pilots based on the students' research projects. The objective was to try to simulate a stroke case assessment by showing a few animations related to simple evaluable actions. For example arm or leg lifting [Figure 1]. We have evaluated the simulation with the medical community and a group of 5 neurologists from Salford Royal NHS Foundation Trust. Although the pilot applications were implemented successfully, and the novel approach well received by the physicians who participated, the simulations were far from being suitable for the trial because of the absence of a proper procedure in developing, testing and case studies.

During this preliminary testing, feedback regarding the application included a need for better animations. It was clear for each respondent that the game had a good base from which to build upon but it needed more work and animations need to be improved in their performance for medical experts to recognise their use. The variety of the animations was also a problem. As we stated in the previous paragraph, in order to correctly assess a stroke we need to analyse 15 different steps of the assessment and this pilot projects covered only simple actions like arm and leg lift.

\subsection{Future Work}

We are currently working on the implementation of all the 15 steps of the assessment. Each step is designed to 


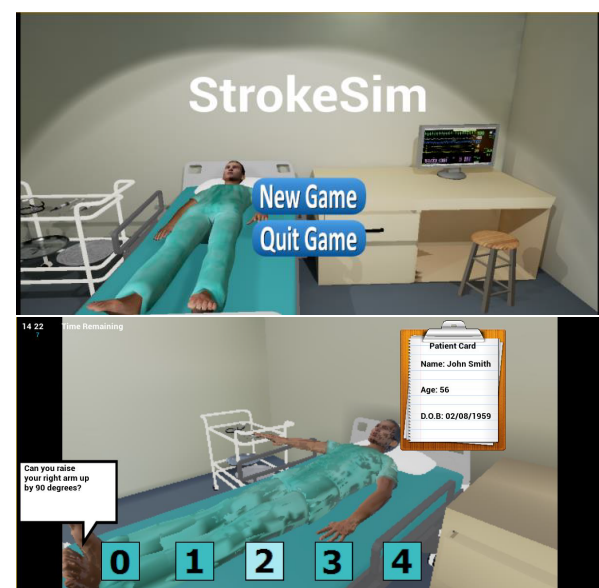

Figure 1: Screenshots from one of the pilot projects. In the second image, the patient is asked to raise the arm to 90 degrees since the movement is not fully achieved the assigned rank for that task is 2 .

be as realistic as possible. The level of realism is important especially for the animations of movement and facial expressions. These features in conjunction with the use of Virtual Reality, are the key milestones of our simulation, which is implemented in the form of a serious game [Figure 2]. Once the game has been released, the next step is to perform various tests aimed at evaluating different aspects of the simulation. We will carry out an evaluation for the VR learning environment to assess the realism and the accuracy of the game, evaluation of the effectiveness end the usability of our tool and evaluation based on the comparison between the results of the standard training and our training method. This last step will be focused on analysing common factors between the two methods like the time spent on each step of the assessment throughout the training, the number of correct results or the number of training sessions needed to learn how to correctly assess the stroke. All those metrics will be considered as important markers needed to affirm or dispute the efficiency of our project as a training tool.

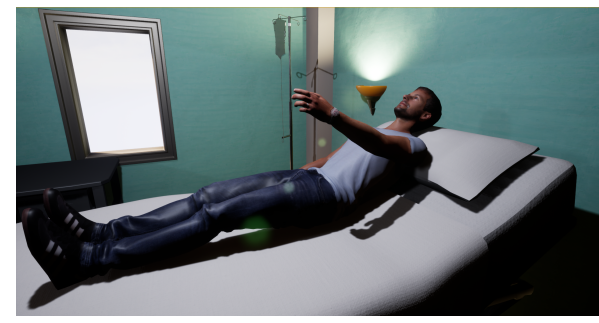

Figure 2: Screenshot from the new concept. The patient is asked to raise the arm to 45 degrees and if he will be capable of keeping the arm straight with no drift for 10 seconds the score for this step of the assessment will be 0 out of 4 , where a higher score means a more severe condition of the patient.

\section{CONCLUSION}

The first part of this paper aimed to conduct a detailed literature survey on the virtual environment and serious games used in healthcare. We listed some existing methods for simulations and serious games used in neurological clinical practice. We explained the concept of Virtual Reality and the differences with Immersive Virtual reality. We also described several publications in the field of Serious Games and finally Serious Games in healthcare. We can affirm that Virtual Reality and Serious Games represent an efficient combination when dealing with educational problems. Their value for learning and rehabilitation purposes is vastly acknowledged and there is still the potential for improvement. Many games are used for stroke rehabilitation but we have not found any example of VR Serious Games for the training of doctors and neurologists in the same clinical field. Based on this necessity and starting from a favourable state of the art on the subject we showed two successful pilot studies and we proposed a possible new solution to the absence of a reliable training tool for the assessment of acute stroke patients.

\section{ACKNOWLEDGEMENT}

We would like to thank Jamie Tomlin and Adam Maxwell for their contribution to the pilot projects and to Salford Royal Foundation Trust for helping with the design and evaluation of the pilot studies.

\section{REFERENCES}

[1] Soysa, Ramesha \& Parthiban, R \& How, Khoo. (2012). Interaction-Oriented System for VITAL Framework. Proceedings - 2012 IEEE 4th International Conference on Technology for Education, T4E 2012. 192-195. 10.1109/T4E.2012.44.

[2] Normand, Vronique \& Babski, Christian \& Benford, Steve \& Bullock, Adrian \& Carion, Stphane \& Frécon, Emmanuel \& Kuijpers, Nico \& Thalmann, Nadia \& Slater, Mel \& Smith, Gareth \& Steed, Anthony \& Thalmann, Daniel \& Tromp, Jolanda \& Usoh, Martin \& Van Liempd, Gidi \& Harvey, John. (1999). The COVEN project: exploring applicative, technical and usage dimensions of collaborative virtual environments.

[3] Nakai, Y \& Kamon, N \& Shibata, Y. (2006). Collaborative Virtual Environment Considering Scalability and Usability. 2006. 121 - 121. 10.1109/MDM.2006.46.

[4] Seo, Jinseok \& Kim, Gerard. (2002). Design for Presence: A Structured Approach to Virtual Reality System Design. Presence. 11. 378-403. 10.1162/105474602760204291.

[5] Noll, S \& Paul, C \& Peters, R \& Schiffner, N. (1999). Autonomous agents in collaborative 
virtual environments. 208 - 215. 10.1109/ENABL.1999.805203.

[6] Kuutti, Kari \& Battarbee, Katja \& Säde, Simo \& Mattelmäki, Tuuli \& Keinonen, T \& Teirikko, T \& Tornberg, A.-M. (2001). Virtual Prototypes in Usability Testing. Hawaii International Conference on System Sciences. 5. 5029. 10.1109/HICSS.2001.926545.

[7] Yen, Benjamin \& Ng, Kenny. (2007). Virtual Objects in Electronic Catalogs: A Human-Computer Interface Issue. Systems, Man and Cybernetics, Part A: Systems and Humans, IEEE Transactions on. 37. 599 - 608. 10.1109/TSMCA.2007.897698.

[8] Drettakis, George \& Roussou, Maria \& Reche Martinez, Alex \& Tsingos, Nicolas. (2007). Design and Evaluation of a Real-World Virtual Environment for Architecture and Urban Planning. Presence. 16. 318-332. 10.1162/pres.16.3.318.

[9] Livatino, Salvatore \& Koffel, C. (2007). Handbook for Evaluation Studies in Virtual Reality. 1 6. 10.1109/VECIMS.2007.4373918.

[10] Rozenn Bouville, Valérie Gouranton, Thomas Boggini, Florian Nouviale, Bruno Arnaldi. \#FIVE : High-Level Components for Developing Collaborative and Interactive Virtual Environments. Proceedings of Eighth Workshop on Software Engineering and Architectures for Realtime Interactive Systems (SEARIS 2015), conjunction with IEEE Virtual Reality (VR), Mar 2015, Arles, France. (hal-01147734)

[11] Corrêa, Ana Grasielle \& Borba, Eduardo \& Lopes, Roseli \& Zuffo, Marcelo \& Araujo, Astolfo \& Kopper, Regis. (2017). User experience evaluation with archaeometry interactive tools in Virtual Reality environment. 217-218. 10.1109/3DUI.2017.7893349.

[12] Gebhardt, Sascha \& Pick, Sebastian \& Leithold, Franziska \& Hentschel, Bernd \& Kuhlen, Torsten. (2013). Extended Pie Menus for Immersive Virtual Environments. IEEE transactions on visualization and computer graphics. 19. 644-51. 10.1109/TVCG.2013.31.

[13] Emma, Lógó \& Peter Hamornik, Balazs \& Köles, Máté \& Hercegfi, Karoly \& Tóvölgyi, Sarolta $\&$ Komlodi, Anita. (2015). Usability related human errors in a collaborative immersive VR environment. 5th IEEE International Conference on Cognitive Infocommunications, CogInfoCom 2014 - Proceedings. 243-246. 10.1109/CogInfoCom.2014.7020454.

[14] Muller, Nicolas \& Panzoli, David \& Michel, Galaup \& Lagarrigue, Pierre \& Jessel, JeanPierre. (2017). Learning mechanical engineering in a virtual workshop: A preliminary study on utilisability, utility and acceptability. 55-62. 10.1109/VS-GAMES.2017.8055811.

[15] Zhang, Kun \& Suo, Jintao \& Chen, Jingying \& Liu, Xiaodi \& Gao, Lei. (2017). Design and Implementation of Fire Safety Education System on Campus based on Virtual Reality Technology. 1297-1300. 10.15439/2017F376.

[16] Vega, Jessica \& Rose, Sophia \& Eckhardt, Christian \& Tahai, Liudmila \& Humer, Irene \& Pietroszek, Krzysztof. (2017). VR wildfire prevention: teaching campfire safety in a gamified immersive environment. 1-2. 10.1145/3139131.3141218.

[17] Andrés, P.M., Serón, F.J., López-Moreno, J., \& Carvalho, C.V. (2014). TimeMesh: Producing and Evaluating a Serious Game. Interacción.

[18] American Institutes for Research. Does deeper learning improve student outcomes? Results from the study of deeper learning: Opportunities and outcomes. August 2016. Retrieved https://www.air.org/sites/default/files/DeeperLearning-Summary-Updated-August-2016.pdf

[19] Southgate, Erica. (2019). Virtual Reality for Deeper Learning: An Exemplar from High School Science. 1633-1639. 10.1109/VR.2019.8797841.

[20] L. Zhang, D. A. Bowman and C. N. Jones, "Exploring Effects of Interactivity on Learning with Interactive Storytelling in Immersive Virtual Reality," 2019 11th International Conference on Virtual Worlds and Games for Serious Applications (VS-Games), Vienna, Austria, 2019, pp. 1-8.

[21] Ribeiro, Matheus \& Tori, Allan \& Tori, Romero \& Nunes, Fátima. (2019). Immersive game for dental anesthesia training with haptic feedback. 1-2. 10.1145/3306214.3338592.

[22] Ushaw, Gary \& Sharp, Craig \& Hugill, Jessica \& Rafiq, Sheima \& Black, Carla \& Casanova, Therese \& Vancleef, Kathleen \& Read, Jenny \& Morgan, Graham. (2017). Analysis of Soft Data for Mass Provision of Stereoacuity Testing Through a Serious Game for Health. 216-220. 10.1145/3079452.3079496.

[23] Kaul, Paras. (2006). Neurological gaming environments. 25. 10.1145/1179295.1179321.

[24] Gazihan Alankus, Amanda Lazar, Matt May, and Caitlin Kelleher. 2010. Towards customizable games for stroke rehabilitation. In Proceedings of the SIGCHI Conference on Human Factors in Computing Systems (CHI '10). ACM, New York, NY, USA, 2113-2122.

[25] K. Choi, "Virtual Reality Wound Care Training for Clinical Nursing Education: An Initial User Study," 2019 IEEE Conference on Virtual Reality and 3D User Interfaces (VR), Osaka, Japan, 2019, 
pp. $882-883$.

[26] Bozkurt, Aras. (2017). Gamification, Education and E-learning: An interview with Andrzej Marczewski.

[27] Pastushenko, Olena \& Geurts, Luc \& Hruska, Tomas. (2019). Conceptual Learning of Electric and Electronic Circuits With Gamification. 589596. 10.1145/3341215.3356305.

[28] Coull, Natalie \& Donald, Iain \& Ferguson, Ian \& Keane, Eamonn \& Mitchell, Thomas \& Smith, Oliver \& Stevenson, Erin \& Tomkins, Paddy. (2017). The Gamification of Cybersecurity Training. 108-111. 10.1007/978-3-319-65849-0_13.

[29] Ard Jacobs, Annick Timmermans, Marc Michielsen, Maaiken Vander Plaetse, and Panos Markopoulos. 2013. CONTRAST: gamification of arm-hand training for stroke survivors. In CHI '13 Extended Abstracts on Human Factors in Computing Systems (CHI EA '13). ACM, New York, NY, USA, 415-420. DOI: 10.1145/2468356.2468430

[30] Diehl LA, Souza RM, Gordan PA, Esteves RZ, Coelho ICM. InsuOnline, an Electronic Game for Medical Education on Insulin Therapy: A Randomized Controlled Trial With Primary Care Physicians. J Med Internet Res 2017 Dec 09;19(3):e72 doi: 10.2196/jmir.6944

[31] Gauthier A, Corrin M, Jenkinson J. Exploring the influence of game design on learning and voluntary use in an online vascular anatomy study aid. Computers \& Education 2015 Sep;87:24-34. doi: 10.1016/j.compedu.2015.03.017

[32] Boada I, Rodriguez-Benitez A, Garcia-Gonzalez JM, Olivet J, Carreras V, Sbert M. Using a serious game to complement CPR instruction in a nurse faculty. Comput Methods Programs Biomed 2015 Nov;122(2):282-291. doi: 10.1016/j.cmpb.2015.08.006

[33] https://www.nhs.uk/conditions/stroke/

[34] http://www.nihstrokescale.org/ 